\title{
THE FORMATION OF DISLOCATION STRUCTURE AND NUCLEATION OF RECRYSTALLIZED GRAINS IN AN ALUMINUM SINGLE CRYSTAL
}

\author{
J.F. BUTLER, JR. , M. BLICHARSKI"* and HSUN HU \\ Department of Materials Science and Engineering \\ University of Pittsburgh, Pittsburgh, PA 15261, USA \\ United States Steel Technical Center \\ 4000 Tech Center Drive, Monroeville, PA 15146, USA \\ "Department of Metallurgy, Academy of Mining and Metallurgy \\ 30-059 Krakow, Poland
}

\section{INTRODUCTION}

Recrystallization of a heavily deformed metal occurs by the nucleation of essentially strain free grains and their subsequent growth at the expense of the deformed matrix. These recrystallization nuclei or newly formed grains usually emerge in regions of high lattice curvature, where the orientation changes with a steep gradient from one matrix orientation to another. These regions were found first in a deformed Si-Fe single crystal to be consisted of finely banded structural elements, hence the name transition band (or microbands) was given to these regions[1,2]. Later studies on heavily rolled copper and aluminum polycrystals also found similar regions of large lattice curvature believed to be the sites of nucleation of cube oriented grains[3,4]. However, the spatial distribution of microstructure and orientation which develops in polycrystalline copper and aluminum after large reductions is very complex and has not at all been characterized. This complication adds to the uncertainties over the nature of the nucleation sites for recrystallized grains, and in particular for the formation of the cube oriented recrystallized grains in heavily deformed copper and aluminum that develops a strong cube texture upon recrystallization.

The present investigation was undertaken in an attempt to give further understanding on the origin of the cube texture in fce metals. An aluminum single crystal with an initial orientation of (001)[110] was used. It is known that this orientation is unstable. Upon rolling deformation the crystal rotates about its [110] axis in the transverse direction (TD) toward the (112)[11 $]$ and (112) [ $\overline{11} 1]$ orientations which are characteristic of the copper type rolling texture. The development of the dislocation substructure in connection with textural changes, as a function of strain in channel die compression, and the orientation aspects of nucleation during recrystallization that leads to the formation of the recrystallization texture, have been studied in considerable detail.

\section{EXPERIMENTAL PROCEDURE}

Crystals of dimensions $9.53 \mathrm{~mm} \times 22.23 \mathrm{~mm} \times 250 \mathrm{~mm}$ were grown by the Bridgman method with controlled orientation. The crystal was deformed using a channel-die device to impart homogeneous plane strain compression. Samples for optical metallography and SEM were prepared from the longitudinal plane (section-plane normal to TD). The samples were anodized to reveal the microstructure, and photographed under polarized light. For SEM, the same samples were stripped of the anodized film to produce a distortion free surface. Electron backscattering patterns (EBSP) were used to determine the orientation of individual subgrains. Correspondence between the image and the EBSP was achieved by creating contamination spots on the subgrain of interest[5]. Thin foils were prepared from the longitudinal plane. This plane provided a clear view of the subgrain structure at all levels of strain. This technique was first developed by Hu to provide a clear view of the structure of cold rolled polycrystalline copper[6]. The orientation of local areas was determined by analysis of the Kikuchi lines in the selected area diffraction patterns.

\section{RESULTS AND DISCUSSION}

\subsection{Formation of the Deformed Structure.}

Two distinguishable texture components were first observed after $40 \%$ reduction ( $\epsilon=-$ 0.51) with the pole figures. The texture formed by divergent rotation of regions of the crystal about the transverse direction. Two stable copper type components evolved after a reduction of 
$70 \%(\epsilon=-1.20)$ (Fig. 1) and they remained stable at least to $90 \%$ reduction $(\epsilon=-2.30)$. During the evolution of the texture, the microstructure underwent substantial changes.

At a reduction of $40 \%(\epsilon=-0.51)$ the divergence of the orientation became more extended, and further rotations of the substructure elements were evident (Fig. 2a and 3). The final stable orientation components were developed at $70 \%$ reduction $(\epsilon=-1.20)$, and the structure appears to be continuing the transition from a structure elongated at an angle to the rolling direction to one elongated parallel to the rolling direction (Fig. 2b). With further deformation to $90 \%$ reduction ( $\epsilon=-2.30$ ), a laminated structure develops parallel to the rolling direction (Fig. 2c). The laminated structure can best accommodate the shears which occur in the (112)[11 $\overline{1}]$ and (112) [ $[111]$ orientations. It is in this laminated structure in which the recrystallization studies were carried out.

The evolution of orientation and microstructure can be followed nicely with the SEM using EBSP's and backscattered electron images. Changes in orientation were documented by 15 to 25 EBSP's taken at regular $58 \mu \mathrm{m}$ intervals along a trace parallel to the normal direction. Micrographs of the representative microstructures illustrate the refinement of the subgrain size with increasing reduction (Fig. 3). The formation of two separate components can be seen in the (111) pole figures of the EBSP results. At $20 \%$ and $40 \%$ reduction, the rotation of the orientation occurs exclusively about the transverse direction. Once the stable copper components are developed, there is an additional rotation about the rolling direction. The substructure also changes from coarse elongated subgrains at $20 \%$ reduction to more equiaxed fine subgrains at $70 \%$ reduction. A dramatic decrease in the subgrain size also occurred with increasing deformation. The refinement in subgrain size occurs concurrently with the activation of an additional pair of slip systems. Using the Tucker stress state for plane strain compression, the resolved shear stress can be calculated as a function of rotation about the transverse direction[7]. Fig. 4 shows the change in $\tau / \sigma$ for the three types of slip systems which are active in the deformation of a single crystal with initial orientation (001)[110]. The first and second systems are equivalent as long as the initial orientation remains stable. These are labeled 1 and 2 in Fig. 4 and 5 . Slip planes 1 and 2 are both coplanar slip planes with two directions of slip in each plane. Slip occurs on plane 1 in directions Ia and Ib for the first coplanar system and on plane 2 and in direction IIa and IIb for the second coplanar system. As long as the crystal orientation remains stable, slip should occur equally on all four systems. However, once rotation does occur, the slip will concentrate on that plane which moves closer to the compression axis, 1, and decrease on the plane which moves toward the rolling direction, 2 (Fig. 5). This concentration of slip on one plane is due to an increase in $\tau / \sigma$ for the slip system whose slip direction moves toward the tensile axis (RD) and a decrease in $\tau / \sigma$ for the slip system whose slip direction rotates toward the compression axis (ND). The effect of this slip concentration was observed at $40 \%$ reduction, where the subgrains consisted of boundaries elongated parallel to the active coplanar slip planes in the local region (Fig. 6). The slip on one slip plane, 1, with two slip directions, Ia and Ib, continues with increasing deformation and causes further rotation of the crystal orientation (Fig. 5). The rotation is observed between $20 \%$ and $70 \%$ reduction. With the rotation, $\tau / \sigma$ begins to increase from rero for slip plane 3 (Fig. 4). The resolved shear stress for the collinear system 3 does not equal that of system 1 until the stable copper type component, (112) [11 $\overline{1}]$, is achieved. The primed numbers mark the positions which correspond to the (112)[11 $\overline{1}]$ position. The activation of the collinear systems results in subgrain refinement due to slip through the previously formed elongated structure. The slip effectively cuts up the elongated subgrains developed during coplanar slip by forming subgrain walls on the previously inactive collinear slip planes. Different regions of the crystal rotated in opposite directions and caused formation of two copper components. One sense of rotation is drawn in Fig. 5 for clarity.

Two orientations were shown to develop after reduction of $40 \%$ by both SEM/EBSP and pole figure techniques. The microstructure as viewed in the TEM revealed the presence of transition bands between the matrix deformation bands (Fig. 6). The upper regions of the micrographs shows elongated subgrains parallel to the coplanar slip plane. The [111] vector is marked by the white arrow. The lower region has subgrains elongated in another direction which corresponds to the coplanar slip plane for that region. The intersection between the two regions consists of interpenetrating elongated subgrains which results in nearly equiaxed subgrains in the transition band. Previously, transition bands of this type were seen in copper and ferritic stainless 
steel $[8,9,10,11]$. The previous work on copper and ferritic stainless steel showed a narrowing of the transition band as strain increases. The elongation of the subgrains remains parallel to the slip plane, but the width of the transition band decreases to a high angle boundary. In the present study, reduction above $40 \%$ produced a structure with roughly equiaxed and approximately equalsized subgrains and the degeneration of the transition band to a high angle grain boundary. These morphological changes in microstructure are apparently a consequence of dynamic/static recovery.

After $70 \%$ reduction, the final complementary copper orientations, (112)[11 1$]$ and (112)[111] are stable. The refinement of the subgrain size was previously shown in Fig. 3 . In the TEM, the refinement of the subgrain size was also observed and is accompanied by a degeneration of the transition bands into high angle grain boundaries. Such a feature is seen in Fig. 7. The letters AA and BB indicate the high angle boundaries which separate regions of complementary orientation. The [111] coplanar slip plane normal for each region is denoted with a white arrow. 3.2. Nucleation and Growth of Recrystallized Grains.

The recrystallization texture produced after annealing for five minutes at $200^{\circ} \mathrm{C}$ was a rotated cube texture (Fig. 8). The ideal orientations can be described as (001)[410] and (001)[410] which are both about $14^{\circ}$ away from the ideal cube orientation, (001)[100]. Annealing below the recrystallization temperature provided an opportunity to observe the formation of nuclei prior to their growth into the surrounding matrix (Fig. 9). The subgrain labeled $A$ has a size advantage over the surrounding subgrains and possesses a large misorientation to the surrounding matrix. Subgrain A has the ability to increase in size by combining with subgrain B since only a small angle boundary separates the two subgrains. If by the process of coalescence the boundary between $A$ and $B$ could be dissolved, a subgrain of almost twice the size of $A$ with a similar misorientation to the surrounding matrix would be formed. Another aspect of the region of enlarged subgrains in Fig. 9. is that the nuclei do not lie at a "transition boundary" between the two complementary copper components. On one side of the subgrain is the orientation (211)[111] and on the other side is a subgrain with the orientation (833)[688]. These two orientations are close to each other and are only separated by a $7.3^{\circ}$ rotation about the transverse direction. In other examples, nuclei were found to have the same component of the copper orientation both the upper and lower sides. However, within several subgrain spacings usually a "transition boundary" was found. A (111) pole figure of the nuclei orientations are shown in Fig. 10. These nuclei orientations can be grouped into two sets: one set very close to the cube position and the other set at an intermediate point between the cube and one of the copper texture components. The exact reason for the formation of the rotated cube texture is not known, but its formation could be due to the lack of polycrystalline features. The polycrystalline rolling texture possesses more orientations of which one is the $S$ type. The $S$ components, $\{123\}<412>$, could provide regions into which the exact cube grains could grow with some advantage. As shown in the growth of the nuclei, there exists the fast growth misorientation between the $\{111\}$ poles of the collinear slip planes and the rotated cube( Fig. 11). The cube grains which were also found in the unstable crystal did not appear to grow because of an unfavorable orientation relationship with the deformed matrix, but if the $\mathbf{S}$ components were present the exact cube grains would have a region to grow into with some advantage. In the unstable single crystal, the constraints from other grains are not as complex as in the polycrystalline aluminum. The additional constraints found in polycrystalline material could act to produce the exact cube. It should be noted that although there are only seven nuclei plotted, they tended to occur with a frequency of well less than one per TEM foil. This is an indication of the rarity of nucleation at the very early stages of recrystallization conducted in the present study. The nucleation in aluminum must be due to the abnormal growth of certain subgrains into viable nuclei, but unfortunately the activation of the collinear slip systems has refined the elongated subgrains to a size nearly equal that of the subgrains in the transition bands.

Figure 11 shows an isolated recrystallized grain as viewed in the SEM. The orientation of this grain is within the spread of the recrystallization texture. Also, this grain possesses a near fast grow misorientation with the copper components. The coincidence of the rotation axis are approximately $10^{\circ}$. Adjacent to either side at the thickest portion is the same component of the copper orientation. At the extreme tip of the recrystallized grain, the grain was found to lie at a boundary between the two copper components. While it is not possible to get the image resolution of the TEM in the SEM, it is possible to come very close to the diffraction resolution 
of selected area diffraction with the EBSP technique. The original position of the nuclei relative to the "transition boundary" is not known, but the recrystallized grain has its origin very close to the boundary between the complementary copper orientations.

\section{CONCLUSIONS}

1. The instability of the crystal orientation (001)[110] in plane strain compression in the channel-die results in the formation of the stable copper components by rotations about the transverse direction. The rotation is accomplished via slip on one of the two initially active coplanar slip systems.

2. The activation of the collinear slip systems once the stable orientation is reached results in the refinement of the elongated subgrains which developed during slip on only the coplanar systems.

3. The transition bands which were observed at low strains (40\%) degenerate into high angle boundaries with increased strain (70\%). The actual mechanism of this microstructural change is not clear. It may likely be a consequence of dynamic/static recovery. The general refinement of the matrix subgrains which appeared to have been resulted from the activation of the collinear systems, may have also made the transition band indistinguishable from the matrix.

4. Nucleation of recrystallized grains was found to occur in regions of apparently homogeneous orientation, but slightly displaced from a "transition boundary." The growth appears to be due to subgrain coalescence.

5. The orientation of the limited number of nuclei observed were within the spread of the recrystallization texture. This suggests that the nucleation limited the possible orientations of the recrystallized grains. Larger $10 \mu \mathrm{m}$ to $40 \mu \mathrm{m}$ diameter grains were observed to possess a near fast growth misorientation to the deformation texture components. These were the grains which contributed to the rotated cube recrystallization texture. It appears that both nucleation and growth contributed to the formation of the rotated cube in the single crystal studied. This texture has also been observed in coarse grained aluminum after rolling and recrystallization.

6. The reason for the formation of the rotated cube recrystallization texture is not known, but it may be due to the simplistic structure formed during deformation. Polycrystalline deformation structures possess more texture components of which one is the $\mathrm{S}$ component. The cube nuclei formed in deformation bands of copper components would have a growth rate advantage over non cube grains if the $S$ type components were present near the nucleation sites.

\section{ACKNOWLEDGEMENTS}

The authors would like to thank the National Science Foundation for their support under grant DMR-8614903. JFB would also like to thank Alcoa Laboratories for their support. Alcoa Laboratories also provided the SEM/EBSP facilities and deserves thanks.

\section{References}

1. H. Hu, in Recovery and Recrystallization of Metals, ed. H. Himmel, Interscience Publishers, New York, 1962, p. 311.

2. J. L. Walter and E. F. Koch, Acta Met., 11 (1963) p. 923.

3. A. A. Ridha and W. B. Hutchinson, Acta Met., 30 (1982) p. 1939.

4. A. L. Dons and E. Nes, Mat. Sci and Tech.,

5. J. Liu, Alcoa Laboratories, Personal Communication, p. 1989.

6. H. Hu, in Textures in Research and Practice, ed. by J. Grewen and G. Wassermann, Springer, Berlin (1969) p. 200.

7. G. E. G. Tucker, Acta Met., 12(1964) p. 1093.

8. P. J. Wilbrandt and P. Haasen, Z. Metall., $\underline{71}$ (1980) p. 273.

9. J. Baker and J. W. Martin, Met. Sci., 17 (1983) p. 459.

10. S. Dymek and M. Blicharski. Scripta Met., 18 (1984) p.99.

11. M. Wrobel, S. Dymek, M. Blicharski and S. Gorczyca, Tex. and Micro., 10(1988) p. 67. 


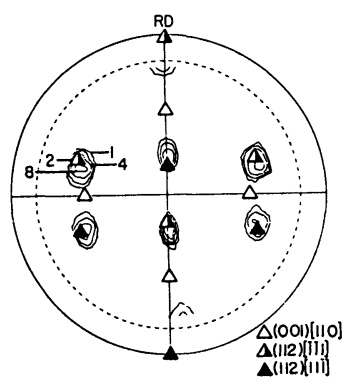

1. (111) pole figure of an aluminum single crystal with initial orientation (001)[110] after $70 \%$ reduction by channel-die compression.
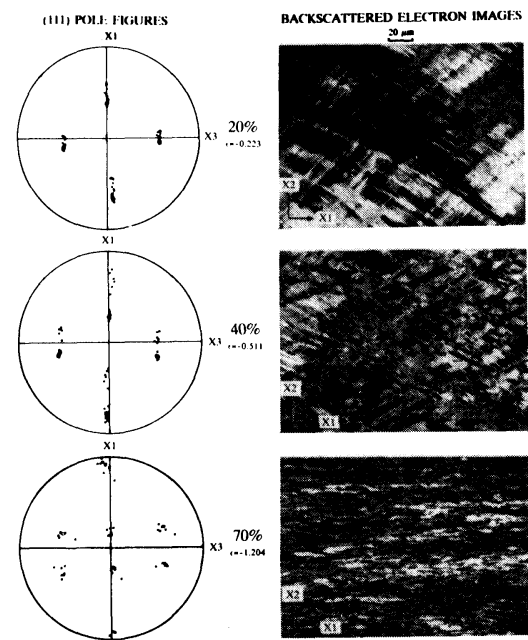

3. Evolution of texture and microstructure with increasing channel-die compression (111) pole figures represent electron backscattering pattern results as taken at $58 \mu \mathrm{m}$ intervals parallel to the normal direction.

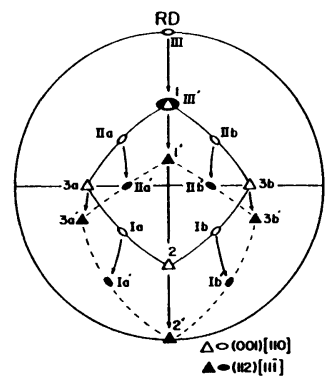

5. Slip systems operating in single crystals of orientations (001) [110] and (112)[111].

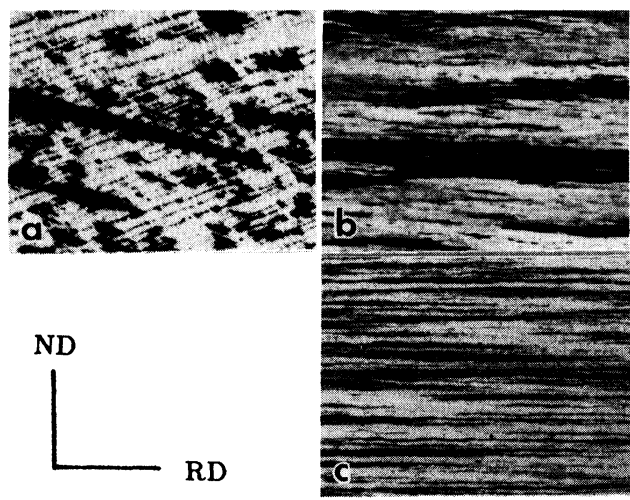

2. Optical micrographs of

$40 \%$ reduction b) $70 \%$ and c) $90 \%$ reduction by channel-die compression.

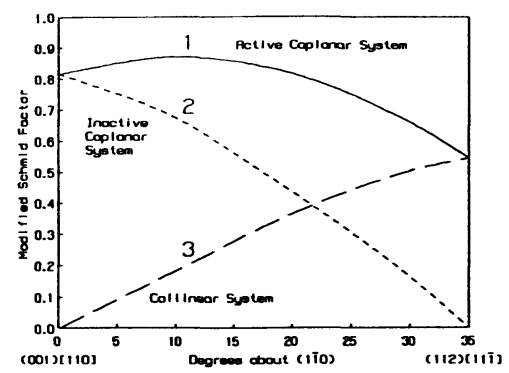

4. Modified Schmid factor, $\tau / \sigma$, as a function of rotation angle about the transverse direction for an F CC crystal with slip systems of the type $\{111\}<110\rangle$ and initial orientation (001) $[110]$.

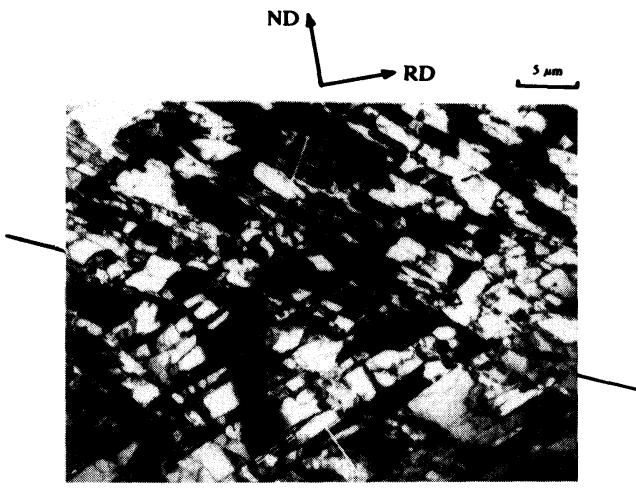

6. TEM micrograph after $40 \%$ reduction by channel-die compression. 


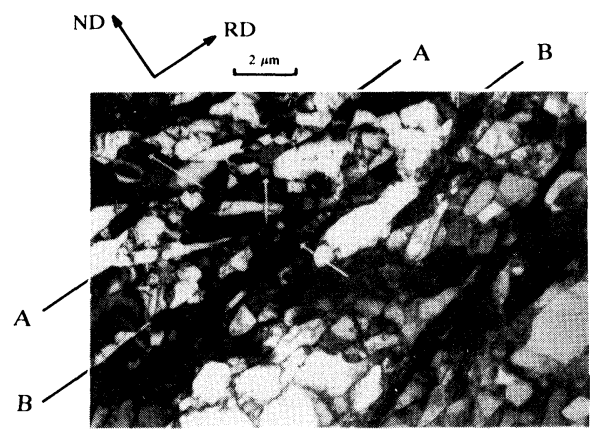

7. TEM micrograph after $70 \%$ reduction by channel-die compression. The transition bands found at $40 \%$ have degenerated into high angle grain boundaries.

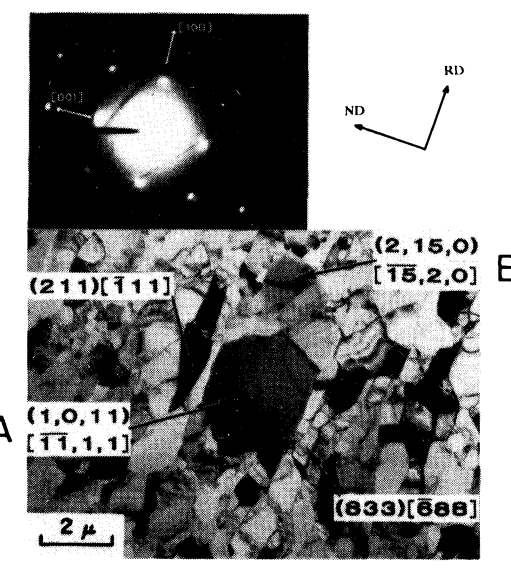

9. TEM micrograph of aluminum single crystal (001) [110] after 90\% reduction and annealing at $100^{\circ} \mathrm{C}$ for five minutes.

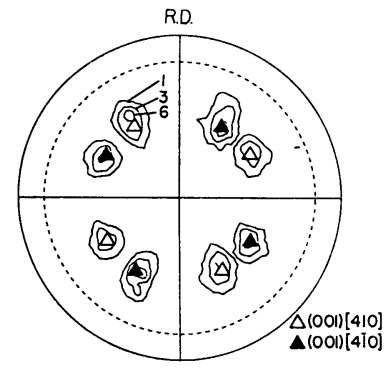

8. (111) pole figure of fully recrystallized single crystal (001) [110] af ter 90\% reduction by channel-die compression and subsequent annealing at $200^{\circ} \mathrm{C}$ for five minutes.

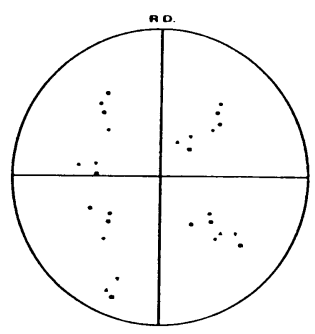

10. (111) pole figure representation of the nuclei orientations observed in samples annealed at $100^{\circ} \mathrm{C}$ for five minutes after $90 \%$ reduction.

11. SEM micrograph of aluminum single crystal (001) [110] after 90\% reduction and annealing at $150^{\circ} \mathrm{C}$ for five minutes after $90 \%$ reduction. The numbers on the micrograph correspond to the numbered (111) poles.

(111)

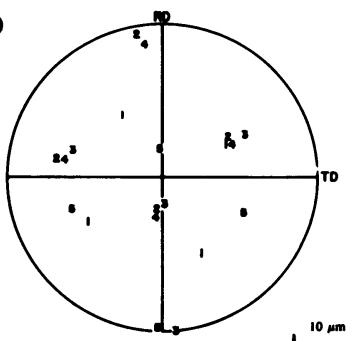

$10 \mathrm{~mm}$

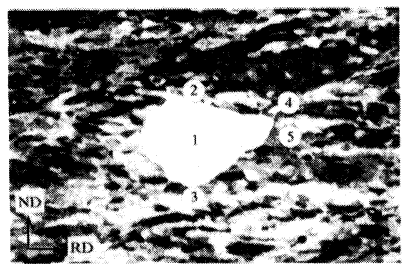

